\title{
Swarm Intelligence Based Dynamic Routing and Wavelength Assignment for Wavelength Constrained All-Optical Networks
}

\author{
Ali Hassan*, Chris Phillips ${ }^{*}$ and Zhiyuan Luo ${ }^{\dagger}$ \\ *Department of Electronics Engineering \\ Queen Mary, University of London, E1 4NS, London-UK \\ E-mail: ali.hassan@elec.qmul.ac.uk \\ †Dept. of Computer Science \\ Royal Holloway University of London, TW20 0EX, Surrey-UK
}

\begin{abstract}
This paper examines the problem of Dynamic Routing and Wavelength Assignment (DRWA) in Wavelength Division Multiplexed (WDM) networks with the wavelength continuity constraint applied. Traditional search algorithms like Integer Linear Programming and Graph Colouring become inefficient for solving optimization problems like DRWA because of the complexity of the task. Particle Swarm Optimization (PSO) inspired by swarm intelligence is proposed here to solve DRWA. A novel fitness function is designed for members of the swarm population that takes into account the normalized path length of the chosen route and the normalized number of free wavelengths available over the whole path. This normalization enables the approach to be self-tuning. Simulation results obtained for NSFNET and EON show that the proposed PSObased scheme achieves a low blocking probability as compared to other swarm intelligence schemes like Genetic Algorithms (GA) for DRWA.
\end{abstract}

Index Terms-Dynamic RWA, Swarm Intelligence, Wavelength Continuity, All-Optical WDM networks.

\section{INTRODUCTION}

Wavelength Division Multiplexed optical networks possess considerable bandwidth capacity [1] as separate optical channels can coexist on the same fibre link. Users communicate by optical channels called 'lightpaths' being setup between the provider-edge access points over a meshed network of links connected via Optical Cross Connect (OXC) devices. However, in order to route information along paths over these links generally requires a process call OpticalElectrical-Optical (O-E-O) conversion inside the OXC devices so that the information can be interrogated and remodulated onto a different wavelength for each leg of its journey. In all-optical networks this process can be simplified allowing the information arriving at the $\mathrm{OXC}$ to remain encoded onto a particular wavelength that is simply redirected to the appropriate output, typically using Micro-ElectroMechanical Systems (MEMS).

The establishment of lightpaths creates logical circuits on top of the physical topology of the WDM optical network. If the intermediate nodes along the chosen route for the lightpath to be setup are not equipped with wavelengthconversion capability [2], then the same wavelength needs to be employed along its entire path. This restriction is known as the wavelength continuity constraint. Furthermore, two lightpaths sharing a common edge (i.e. link) of the network need to be assigned unique wavelengths as shown in Figure 1. In this case two lightpath share a common edge between node ' 7 ' and node ' 10 ', therefore each needs to be assigned a unique wavelength. This is called the "wavelength clash constraint".

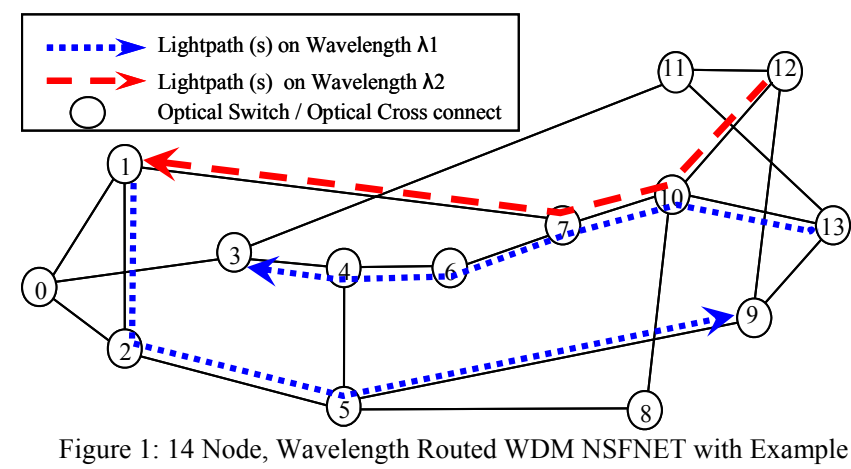

Lightpath Connections

When a connection request is made to setup a new end-toend lightpath, a Routing and Wavelength Assignment (RWA) mechanism attempts to find an appropriate route and assigns a wavelength for it. The RWA approach can be categorized into two types: static and dynamic [3]. In Static RWA (SRWA), all the lightpath requests are known in advance, and the problem is to assign routes and wavelengths in a global manner, while minimizing the network resources [4]. In Dynamic RWA (DRWA), the lightpath requests arrive unexpectedly with random holding times. The objective of DRWA is then to find route and assign wavelength so that the blocking probability of the connection requests is reduced. SRWA is a well-known NP (Nondeterministic Polynomialtime) - Hard problem [5]. DRWA is more challenging because the lightpath requests arrive randomly and stay in the network for random amount of time. Traditional mathematical search schemes like Integer Linear Programming (ILP) and Graph Colouring become ineffective at solving the DRWA problem because of the computational complexity. Therefore, different heuristic and stochastic schemes are used to solve DRWA in a reduced amount of time. Most of the heuristic 
schemes used for DRWA simplify the problem domain by decomposing it into two sub-problems: the routing subproblem and a wavelength assignment sub-problem [4].

Three major routing schemes are: fixed routing, fixedalternative routing and adaptive routing. The simplest among these is fixed routing, where a single route is computed offline for each possible source-destination pair. Whenever a lightpath request arrives, that pre-computed route is always chosen for wavelength assignment. In fixed-alternative routing, a set of routes are pre-computed for each sourcedestination pair and for a given lightpath request, one route is chosen from that set. In the case of adaptive routing, for a lightpath request, a route is computed on-the-fly (dynamically) based on the current network state. Fixed routing is simple to implement, but leads to a high blocking probability. Fixed-alternative routing shows better performance compared to fixed routing. Though computationally expensive as compared to the other two, adaptive routing obtains best performance in terms of blocking probability $[3,4]$.

For wavelength assignment, a number of heuristic methods have been proposed like First-Fit, Least-Used, Most-Used, Min-Product, Max-Sum, Relative-Capacity-Loss (RCL) and so forth. A detailed review of routing of wavelength assignment can be found in [4].

In this paper, a novel particle swarm optimization (PSO) based scheme inspired by swarm intelligence is proposed to provide DRWA under the wavelength continuity constraint. The First-Fit (FF) algorithm is chosen for wavelength assignment, as it is simple to implement as compared to schemes like Max-Sum and RCL. Also the performance difference among various wavelength assignment schemes is not very large [6]. The rest of the paper is organized as follows: Section II introduces the concept of Particle Swarm Optimization (PSO) and the proposed PSO based scheme for DRWA. Section III presents the simulation results and analysis. Finally conclusion is presented in Section IV.

\section{PARTICLE SWARM OPTIMIZATION (PSO)}

\section{A. General PSO Algorithm}

PSO is a population based optimization technique, developed by Kennedy and Eberhart [7, 8], inspired by social behaviour of bird flocking (and schools of fish).

In PSO, a swarm is a collection of particles where each particle has both a position and velocity. The position of the particle represents a candidate solution to the problem space while the velocity is used to move the particle from one position to another. The "classical" PSO equation where the position and velocity represents physical attributes of the particles is represented by (1) and (2).

Calculating a Single Particle's New Velocity:

$$
V_{i d}=V_{i d}+\eta_{1} r_{1}\left(P_{i d}-X_{i d}\right)+\eta_{2} r_{2}\left(P_{i d}^{i}-X_{i d}\right) \quad i=1,2, . ., N . d=1,2, . . D .
$$

"Moving" a Single Particle in a Swarm

$$
X_{i d}=X_{i d}+V_{i d}
$$

$P_{i d}$ is the personal best position, a particle has reached; $\mathrm{P}_{\mathrm{id}}{ }^{\mathrm{n}}$ is the global best position of all the particles. $\eta_{1}$ (the selfconfidence factor) and $\eta_{2}$ (the swarm-confidence factor) are positive constants called 'acceleration constants' to determine the influence of $P_{i d}$ and $P_{i d}{ }^{n} ; r_{l}$ and $r_{2}$ are independent random numbers in the range $[0,1]$. $\mathrm{N}$ is the total number of particles in the swarm and $\mathrm{D}$ is the dimension of the problem search space.

PSO starts by randomly initializing the position and velocities of all the particles in the swarm over the problem space. The position of $\mathrm{i}^{\text {th }}$ particle is represented by the vector $X_{i}=\left[X_{i 1}, X_{i 2} \ldots X_{i D}\right]$ and velocity of $i^{\text {th }}$ particle is represented by the vector $V_{i}=\left[V_{i 1}, V_{i 2} \ldots V_{i D}\right]$, where $D$ is the number of function parameters being optimized. For each iteration (until the convergence criteria is met), the fitness function is applied to the particles to quantize their respective positions over the problem search space. The particle with the best fitness value in the neighbourhood is marked as the global/local best particle. Each particle will also keep a record of its personal best position searched so far. Equation (1) is used to calculate new velocity for each particle in the swarm based on particle's previous velocity, its current and personal best position, and the position of the particle with best fitness value in the neighbourhood. Equation (2) is then used to apply the velocity to the particle. As a result of this, the particle will move to a new position i.e. it will now represent a new candidate solution to the problem being studied.

\section{B. Related Work}

Evolutionary optimization schemes like genetic algorithms (GA) and PSO have successfully been used in the past to solve many NP-hard optimization problems [9]. GA and PSO are similar in the way that both techniques are populationbased search schemes that mimic the natural biological evolution and/or the social behaviour of species $[9,10]$. Each member of the population represents a candidate solution to the problem addressed, and over time they evolve to represent some other candidate solution. One advantage of PSO over GA is that PSO is more computationally efficient [11]. Some performance comparison studies between GA and PSO have been reported in $[9,11,12,13]$.

In [6], a novel GA based scheme is proposed to solve dynamic RWA problem in wavelength routed optical networks. Genetic algorithms are swarm intelligence inspired search schemes based on the idea of natural selection and natural genetics. In [6], a member of the population (gene) represents a route from source to destination node i.e. a candidate solution to the routing sub-problem for DRWA. Genetic operators like crossover, mutation and then selection are applied to create a new population of genes. Ammar et al [14] have proposed a novel hybrid algorithm based on PSO and a noising meta-heuristic for computing shortest paths in the network. The hybrid PSO based scheme shows better performance as compared to GA-based search algorithms for optimal shortest path computation [14]. In [3] and [6], GA algorithms are proposed for solving DRWA in all-optical WDM networks. In our paper, the GA based schemes proposed in [3] and [6] are selected as the schemes for 
performance comparison purposes with our novel PSO-based algorithm.

\section{Proposed PSO Scheme}

This section describes the proposed PSO-based scheme for solving the DRWA problem for all-optical networks with the wavelength continuity constraint applied.

Encoding and Decoding of Particles:

A typical encoding scheme for path representation is the 'direct-representation' scheme [14] where a path is represented as a sequence of node identification numbers from the source node to the destination node. Encoding schemes based on direct representation have been used to encode paths in [15]. Gen et al [16] proposed an indirectrepresentation scheme (priority-based encoding) for solving the shortest path problem using GA. In the priority-based encoding scheme, a path (chromosome) is represented by encoding some guiding information about a node instead of the node-ID. An example of such guiding information can be the node priority. This guiding information is used to generate a path from an arbitrary chromosome. In [17] a 'weighted encoding scheme' is used for chromosome representation in GA, whereas in [14], a cost-priority based encoding scheme is used for representing a particle in PSO.

In this paper, for simplicity, a priority based encoding scheme is used. The position of the particle is represented as a vector of node priorities. The path, which a particle represents, is decoded using a path growth procedure [16] by starting from the source node and then sequentially appending the intermediate nodes one-by-one, till the destination node is reached. During the path growth procedure, if more than one node is available, the node with the highest node priority is selected. Every time as node is selected during path construction, it is marked as unavailable for the rest of path growth procedure. Figure 2 illustrates an example of priority based encoding, where a path is being constructed for a lightpath request between source node ' 1 ' and destination node ' 9 ' in NSFNET (previously shown in Figure 1) by decoding the position of the particle using the 'path growth' procedure.

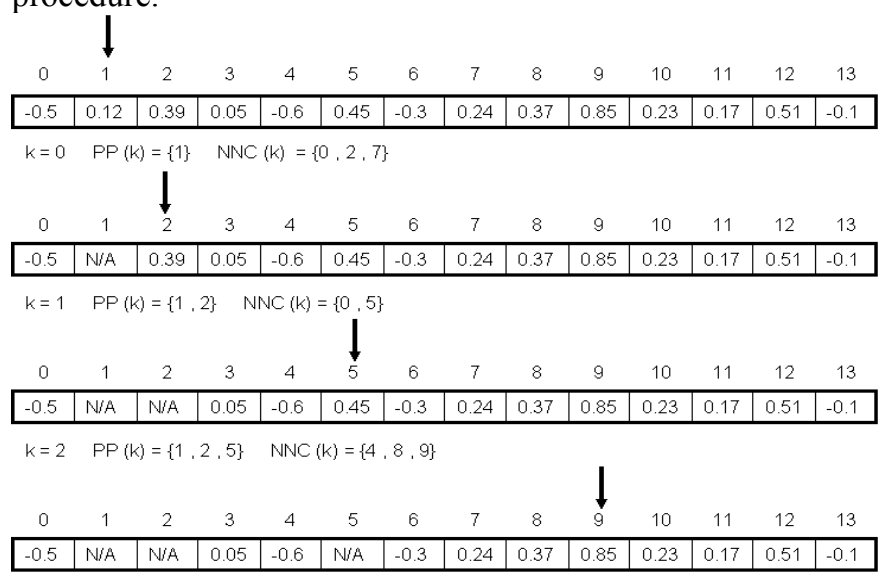

$k=3 \quad P P(k)=\{1,2,5,9\} \quad N N C(k)=0$

Figure 2: Priority based encoding and Path growth procedure for decoding. $\mathrm{k}$

$=$ iteration number. $\mathrm{PP}(\mathrm{k})$ is the 'partial path vector' at iteration ' $\mathrm{k}$ '. $\mathrm{NNC}(\mathrm{k})$ is the 'next node candidate' vector.
Neighbourhood topology:

In PSO, the particle's movement through the search space is influenced by the particle's own experience and the experience of the most successful particle in its neighbourhood. A neighbourhood is a set of particles in the swarm whose positions will influence a particle's own search due to their proximity to the particle. In this paper, a global neighbourhood is used where all the members in the swarm are particle's neighbours.

\section{Swarm initialization:}

In the swarm, 'p' particles are created. Each particle's position (node priorities) and the velocity are randomly initialized with real numbers in the range $[-1.0,1.0]$. In order to avoid the velocity from becoming very large in the initial PSO iteration, and to avoid premature convergence to a local optimum as well as to restrict the influence of particle's old velocity on the current velocity, a number of improvements are suggested in the literature $[18,19,20,21]$. For example, Clerc [22] proposed the use of constriction factor $\chi$ in order to prevent large velocity values.

Therefore, (3) is used in this paper to compute new velocity for a particle.

$V_{i d}=\chi\left[V_{i d}+\eta r_{1}\left(P_{i d}-X_{i d}\right)+\eta_{2} r_{2}\left(P_{i d}^{n}-X_{i d}\right)\right] i=1,2, . ., N . d=1,2, . D$.

$\chi=2\left(\left|2-\eta-\sqrt{\eta^{2}-4 \eta}\right|\right)^{-1} \quad$ if $\eta=\eta_{1}+\eta_{2}>4$

Fitness Function:

In this paper, a novel fitness function is used which takes into account not only the normalized length of the route i.e. number of hops between the source and destination node. It also considers the normalized number of free wavelengths available over the whole route while satisfying wavelength continuity constraint. If no free wavelength is available for the route selected, then the fitness function will assign a large negative fitness value $(-100.0)$ for that particle.

$L_{\max }$ is the maximum length of the route between any source - destination pair and $L_{s d}$ is the length of the route between source ' $s$ ' and destination ' $d$ '. $\alpha[0,1]$ is a design parameter, and $W_{\text {Total }}$ is the total number of wavelengths supported by the optical network. $W_{s d}$ then defines the number of free wavelengths available over the route between source ' $s$ ' and destination ' $d$ '. The fitness function used in this paper is then represented by (5).

$$
F(i)=\left[\alpha * \frac{\left(L_{\max }-L_{s d}\right)}{L_{s d}}\right]+\left[(1-\alpha) *\left(1-\frac{\left(W_{\text {Total }}-W_{s d}\right)}{W_{s d}}\right)\right]
$$

\section{SiMULATION RESULTS AND ANALYSIS}

To evaluate the dynamic RWA performance of the proposed scheme (hereafter simply referred as the PSO algorithm) in terms of blocking probability, a simulator has been implemented in Opnet Modeler ${ }^{\mathrm{TM}}$ [23]. Experiments are conducted for NSFNET and EON networks shown in the 
Figure 1 and 3 respectively, where all WDM links are either assumed to have a capacity of 8 and 16 wavelengths, as indicated.

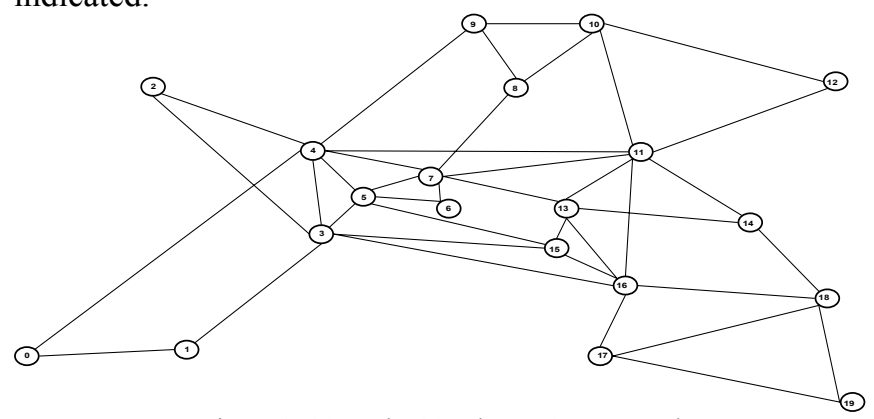

Figure 3: 20 Node, 39 Edges EON Network.

To compare the performance of proposed PSO algorithm, the Genetic Algorithms proposed in [6] and [3] are employed (hereafter simply referred to as GA1 and GA2 algorithms respectively) as well as a shortest path algorithm with first-fit wavelength assignment (hereafter simply referred to as SP FF algorithm).

A dynamic traffic model is used where connection requests were generated at each node following a Poisson process with an arrival rate of $\lambda_{\text {nodes }}$. Destination nodes for the connections are randomly chosen according to a uniform distribution. Therefore, the total connection arrival rate $\left(\lambda_{\text {total }}\right)$ in the whole network is the product of total number of nodes in the network and $\lambda_{\text {nodes }}$.

If $\varphi$ is the total number nodes in the network, then

$\lambda_{\text {total }}=\varphi^{*} \lambda_{\text {nodes }}$

The connection holding time is exponentially distributed with mean ' $T$ ' seconds. Therefore, the normalized traffic load is given by (7).

Normalized Traffic Load $=\lambda_{\text {total }} * T / \varphi *(\varphi-1)$

For the experiments, the value of ' $\mathrm{T}$ ' is kept constant at 50 seconds and the value of $\lambda_{\text {nodes }}$ is changed in order to vary the value of normalized traffic load. A distributed control system is used where each node in the network upon connection request arrival performs a dynamic routing and wavelength assignment computation (using out-of-band signalling). For the wavelength assignment, DIR (destination-initiated reservation) [24] along with a first-fit algorithm is used. This requires three control packets to be sent along the chosen route for connection establishment; one for checking wavelength availability, the second for wavelength reservation and the third for confirmation of the lightpath establishment. Each node is assumed to have wavelength usage information of the whole network. No alternative routing is used and no re-attempts are made for route recomputation. So if the chosen route cannot find a free wavelength, the connection will be blocked. For different traffic loads, each experiment is carried out 10 times with different seeds for the random number generator and the mean values are plotted in the figure 4, 5, 6 and 7 along with the $95 \%$ confidence intervals.

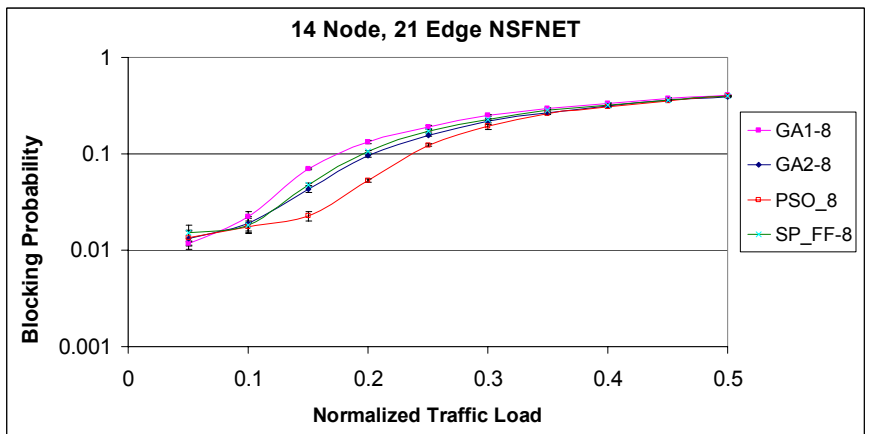

Figure 4: Blocking Probability Versus Normalized Traffic load between PSO, GA1, GA2 and SP_FF algorithms for NSFNET (Figure 1). Number of Wavelengths $=8, \alpha=0.9$, Confidence Interval $=95 \%$, Population size (for both GA and PSO algorithms) $=15$, Iterations $(\mathrm{PSO})=20$, Generations $(\mathrm{GA})$ $=20$.

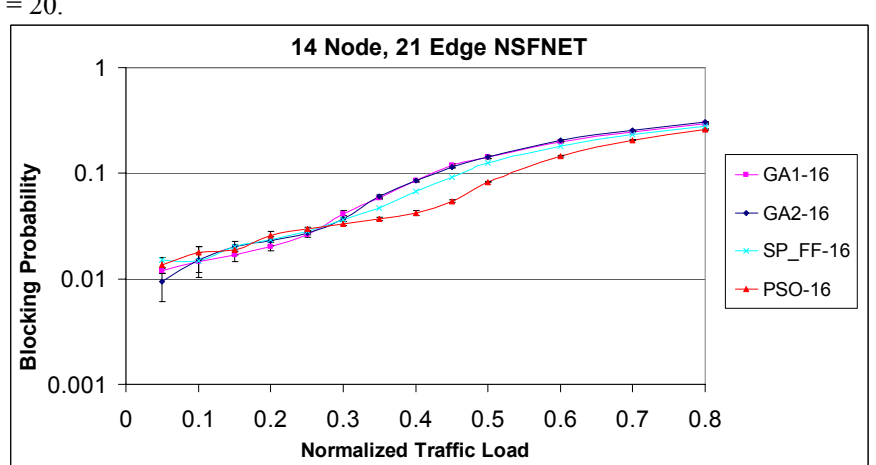

Figure 5: Blocking Probability Versus Normalized Traffic load between PSO, GA1, GA2 and SP_FF algorithms for NSFNET (Figure 1). Number of Wavelengths $=16, \alpha=0.9$, Confidence Interval $=95 \%$, Population size (for both GA and PSO algorithms) $=15$, Iterations $(\mathrm{PSO})=20$, Generations $(\mathrm{GA})$ $=20$

Figure 4 and 5 show the relationship of blocking probability versus the traffic load for 8 and 16 wavelength variants of the NSFNET network respectively. As shown in Figure 4, when the wavelength capacity is 8 per link, all schemes show similar blocking probability performance at low traffic loads i.e. between the normalized traffic loads of 0.05 and 0.1 . When the traffic load is increased i.e. between 0.1 and 0.35 , the PSO scheme shows a significant performance improvement in terms of blocking probability. However, when the traffic loads is increased above 0.4 , both GA and PSO tend to the same blocking performance. The reason is, at very high traffic loads, links become saturated and the availability of free wavelengths becomes very limited. In Figure 5, the number of wavelengths supported by each link is increased to 16 . At low traffic loads i.e. between 0.05 and 0.3 , both GA and PSO schemes show similar blocking probability performance. The reason is, at low traffic loads, these schemes can always find free wavelengths to be assigned whatever route they choose. However, when the traffic load increases to normalized traffic load of between 0.3 and 0.7 , PSO performs significantly better than GA1, GA2 and the SP FF algorithms. Again, as the links saturate in terms of wavelength availability because of already deployed lightpaths over them (i.e. when the normalized traffic load is above 0.7), the PSO scheme shows comparable connection blocking probability performance to the others. 
Similar blocking probability performance behaviour can be observed in Figure 6 and 7, which show the blocking probability versus normalized traffic load for 8 and 16 wavelength based variants of EON network respectively. At very low traffic loads, PSO performs similar to other schemes. Again the reason is that at very low traffic loads, most of the wavelengths are free for the wavelength assignment subproblem. However as the traffic load increases, PSO shows significantly better blocking probability performance as compared to the other dynamic RWA schemes. However, when the links reach saturation at very high traffic loads, all the schemes show similar blocking probability performance, because now most of the wavelengths are already being used in the network.

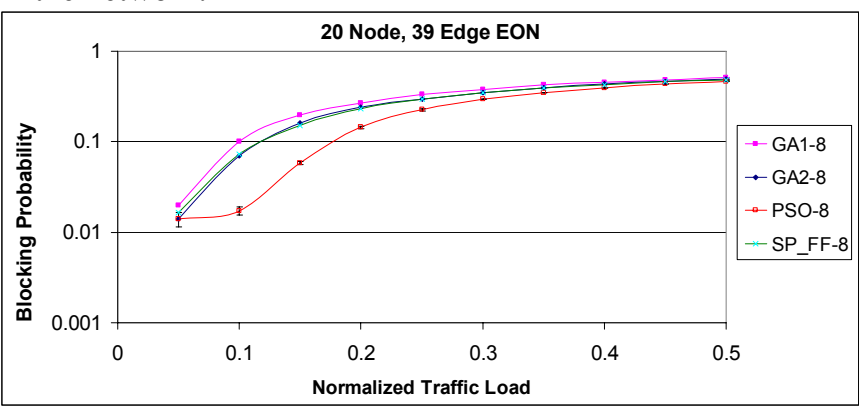

Figure 6: Blocking Probability Versus Normalized Traffic load between PSO, GA1, GA2 and SP FF algorithms for EON (Figure 3). Number of Wavelengths $=8, \alpha=0.9$, Confidence Interval $=95 \%$, Population size (for both GA and PSO algorithms) $=15$, Iterations $(\mathrm{PSO})=20$, Generations $(\mathrm{GA})$ $=20$.

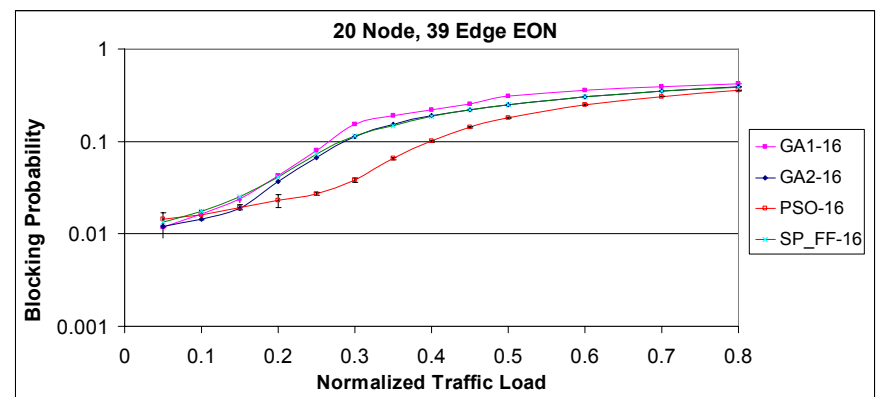

Figure 7: Blocking Probability Versus Normalized Traffic load between PSO, GA1, GA2 and SP_FF algorithms for EON (Figure 3). Number of Wavelengths $=16, \alpha=0.9$, Confidence Interval $=95 \%$, Population size (for both GA and PSO algorithms) $=15$, Iterations $(\mathrm{PSO})=20$, Generations $(\mathrm{GA})$ $=20$.

In the fitness function, the value of ' $\alpha$ ' is used to control the influence of the path length and the number of free wavelengths available. Generally, the number of free wavelengths and route length (number of hops) are correlated as routes having more free wavelengths tend to be shorter routes. Choosing a route with more free wavelengths gives better blocking probability performance. However, it is observed from the experiments that at very low traffic loads, choosing the shortest route instead of routes having large number of free wavelengths gives better performance. The reason is, when the network load is very low, since most of the wavelengths are free, there is a chance that a longer route has more free wavelengths available than the shorter ones. At the later stage this can led to an increase in the blocking probability. Therefore it would appear that there is a need to dynamically control the ' $\alpha$ ' factor that can give more weight to shorter paths when the network load is low and give more weight to 'number of free wavelengths' at moderate and high traffic loads.

However, in the proposed fitness function, both factors (route length and number of free available wavelengths) are normalized. The advantage of using such a fitness function is that it decreases the variation of mean blocking probability for different values of ' $\alpha$ '. This effectively reduces the need to dynamically adjust the ' $\alpha$ ' factor.

The blocking probability performance of the proposed 'normalized fitness function' given by (5), is carried out for different values of ' $\alpha$ ' in Figure 8. Comparison is made with respect to SP_FF scheme.

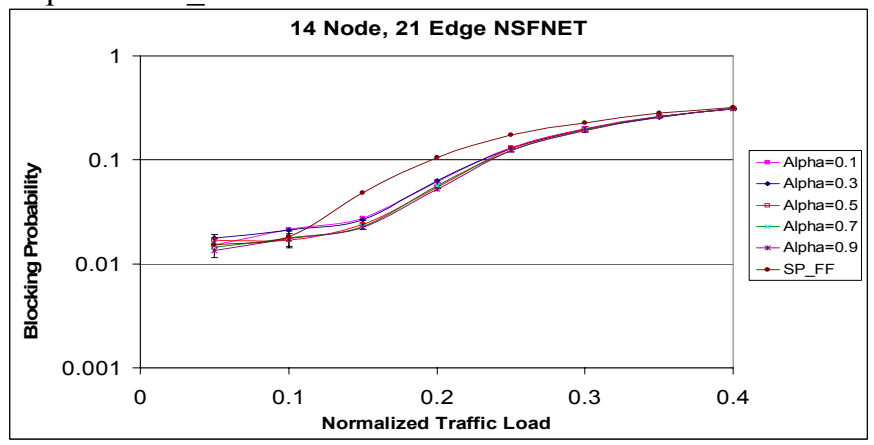

Figure 8: Blocking Probability Versus Normalized Traffic load between different values of ' $\alpha$ ' for PSO using proposed fitness function given by (5) with SP_FF algorithms for NSFNET (Figure 1). Number of Wavelengths $=8$, Population size $=15$, Iterations $(\mathrm{PSO})=20$.

In Figure 9, the same experiment is carried out using a nonnormalized fitness function, as given by (8).

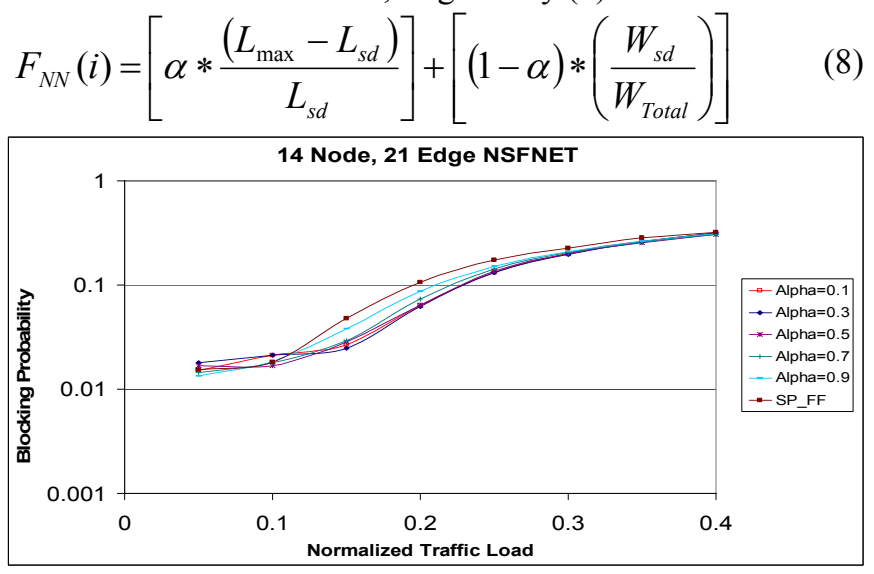

Figure 9: Blocking Probability Versus Normalized Traffic load between different values of ' $\alpha$ ' for PSO using non-normalized fitness function given by (8) with SP_FF algorithms for NSFNET (Figure 1). Number of Wavelengths $=8$, Population size $=15$, Iterations $($ PSO $)=20$.

Figure 8 and 9 clearly show that using the proposed normalized fitness function, reduces the need to dynamically adjust the algorithmic parameter ' $\alpha$ ' as compared to the nonnormalized fitness function case where altering $\alpha$ is seen to have a notable impact on the blocking probability performance.

Generally, the reason that PSO performs better than GA in terms of blocking probability is that, during GA's 
reproduction stage, the selection operator eliminates those members of the population which have a poor fitness value from being included in the next generation. However in PSO, there is no member selection operator. So there is always a chance that a member of population that has a poor initial fitness value, might evolve over time through self-exploration of the search space and by 'exploiting' the exploration of the best member in the neighbourhood, to become the best member of the population.

\section{CONCLUSIONS}

In this paper a new algorithm based on particle swarm optimization (PSO) is proposed, which effectively solves dynamic RWA problem for all-optical WDM networks under the wavelength continuity constraint. The proposed fitness function takes into account normalized path length and the normalized number of free wavelength available during route evaluation. The simulation results show that the PSO algorithm shows better performance in terms of blocking probability as compared to the Genetic Algorithms proposed in [3] and [6] and a Shortest Path - First Fit heuristic algorithm. The normalization of 'path length' and 'number of free wavelengths' effectively reduces the need to have dynamic controlling factor ' $\alpha$ '. For these reasons, the proposed swarm intelligence inspired, PSO scheme is a suitable candidate for dynamic RWA in All-Optical WDM networks.

\section{ACKNOWLEDGMENT}

This work is supported by the EPSRC UK Research Council under Grants EP/D078741/1 and EP/E000053/1, "Machine Learning for Resource Management in NextGeneration Optical Networks".

\section{REFERENCES}

[1] B. Mukherjee, Optical Communication Networks, McGraw-Hill, New York, 1997.

[2] I. Chlamtac, A. Ganz, and G. Karmi. "Lightpath Communications: An Approach to High-Bandwidth Optical WAN's" IEEE Transactions on Communications, vol. 40, no. 7, pp. 1171-1182, July 1992.

[3] Vinh Trong Le et al. "A Genetic Algorithm for Dynamic Routing and Wavelength Assignment in WDM Networks". Publisher Springer Berlin / Heidelberg. ISSN 0302-9743 (Print). Volume 3358/2004. ISBN 978-3-540-24128-7.

[4] Hui Zang et al: "A Review of Routing and Wavelength Assignment approaches for Wavelength-Routed Optical WDM networks". Optical Networks Magazine, vol. 1, (2000) 47-60.

[5] Ramaswami, R., Sivarajan, K.N.: "Routing and Wavelength Assignment in all-optical networks". IEEE/ACM Transactions on Networking, vol. 3 (1995) 489-500.

[6] D. Bisbal et al., "Dynamic routing and wavelength assignment in optical networks by means of genetic algorithms," Photonic Network Communications, vol.7, no.1, pp.43-58, 2004.

[7] Kennedy, J. and Eberhart, R. C. (1995). "Particle swarm optimization". Proc. IEEE Int'l. Conf. on Neural Networks, IV, 1942-1948. Piscataway, NJ: IEEE Service Center.
[8] Eberhart, R. C. and Kennedy, J. "A new optimizer using particle swarm theory" Proceedings of the Sixth International Symposium on Micromachine and Human Science, Nagoya, Japan. pp. 39-43, 1995.

[9] E. Elbeltagi, T. Hegazy, and D. Grierson, "Comparison among five evolutionary-based optimization algorithms," Advanced Engineering Informatics, vol. 19, no. 1, pp. 43-53, 2005.

[10] R. Hassan et. al. "A comparison of particle swarm optimization and the genetic algorithm," in Proceedings of the 1st AIAA Multidisciplinary Design Optimization Specialist Conference, Austin, Tex, USA, April 2005.

[11] D. W. Boeringer and D. H. Werner, "Particle swarm optimization versus genetic algorithms for phased array synthesis," IEEE Transactions on Antennas and Propagation, vol. 52, no. 3, pp. 771- 779, 2004.

[12] C. Mouser and S. Dunn, "Comparing genetic algorithms and particle swarm optimization for an inverse problem exercise," The Australian \& New Zealand Industrial and Applied Mathematics Journal, vol. 46, part C, pp. C89-C101, 2005

[13] R. C. Eberhart and Y. Shi, "Comparison between genetic algorithms and particle swarm optimization," in Proceedings of the 7th International Conference on Evolutionary Programming, pp. 611-616, Springer, San Diego, Calif, USA, March 1998.

[14] A. W. Mohemmed and N. C. Sahoo. "Efficient Computation of Shortest Paths in Networks Using Particle Swarm Optimization and Noising Metaheuristics". Hindawi Publishing Corporation, Discrete Dynamics in Nature and Society, Volume 2007, Article ID 27383, 25 pages, doi:10.1155/2007/27383.

[15] M.Munemoto, Y. Takai, and Y. Sato, "A migration scheme for the genetic adaptive routing algorithm," in Proceedings of IEEE International Conference on Systems, Man, and Cybernetics, vol. 3, pp. 2774-2779, San Diego, Calif, USA, October 1998.

[16] M. Gen, R. Cheng, and D. Wang, "Genetic algorithms for solving shortest path problems," in Proceedings of the IEEE International Conference on Evolutionary Computation, pp. 401-406, Indianapolis, Ind, USA, April 1997.

[17] G. Raidl and B. A. Julstrom, "A weighted coding in a genetic algorithm for the degree constrained minimum spanning tree problem," in Proceedings of the ACM Symposium on Applied Computing (SAC '00), vol. 1, pp. 440-445, Italy, March 2000.

[18] Shi, Y. H., Eberhart, R. C., (1998), A modified particle swarm optimizer, Proc. Of 1998 IEEE International Conference on Evolutionary Computation, Anchorage, AK, in press.

[19] P. Srinivas and R. Battiti, "The gregarious particle swarm optimizer (G-PSO)," in Proceedings of the 8th Annual Conference Genetic and Evolutionary Computation (GECCO '06), pp. 67-74, Seattle,Wash, USA, July 2006.

[20] X.-F. Xie, W.-J. Zang, and Z.-L. Yang, "Dissipative particle swarm optimization," in Proceedings of the Congress on Evolutionary Computation (CEC '02), vol. 2, pp. 14561461,Honolulu,Hawaii, USA, May 2002.

[21] M. Iqbal, A. A. Freitas, and C. G. Johnson, "Varying the topology and probability of reinitialization in particle swarm optimization," in Proceedings of the 7th International Conference on Artificial Evolution, Lille, France, October 2005.

[22] M. Clerc, "The swarm and queen: towards a deterministic and adaptive particle swarm optimization," in Proceedings of the Congress on Evolutionary Computation (CEC '99), vol. 3, pp. 1951-1957, Washington, DC, USA, July 1999.

[23] Opnet Modeler, http://www.opnet.com

[24] Lu, K., Xiao, G. and Chlamtac, I.: Analysis of Blocking Probability for Distributed Lightpath Establishment in WDM Optical Networks. IEEE/ACM Transactions on Networking, Vol. 13, No. 1 (2005) 187-197. 